https://doi.org/10.22319/rmcp.v11i3.5127

Nota de investigación

\title{
Tiempo de manejo y algunas conductas relacionadas con el estrés al manejar grupos grandes o reducidos de ganado en mangas rectas
}

Miguel Ángel Damián ${ }^{\mathrm{a}}$

Virginio Aguirre ${ }^{a}$

Agustín Orihuela ${ }^{\mathrm{a} *}$

Mariana Pedernera ${ }^{a}$

Saúl Rojas ${ }^{b}$

Jaime Olivares $^{\text {b }}$

${ }^{a}$ Universidad Autónoma del Estado de Morelos. Facultad de Ciencias Agropecuarias. Avenida Universidad 1001 Colonia Chamilpa, 62210, Cuernavaca, Morelos. México.

${ }^{\text {b }}$ Universidad Autónoma de Guerrero. Facultad de Medicina Veterinaria y Zootecnia, Cd. Altamirano, Guerrero. México.

* Autor de correspondencia: aorihuela@uaem.mx

\section{Resumen:}

El objetivo del presente estudio fue evaluar el tiempo de manejo y el comportamiento indicativo de estrés en bovinos durante su paso por una manga recta. Se utilizaron ocho hatos de 50 animales Brahman x Pardo Suizo, cada hato constituido proporcionalmente por animales jóvenes, adultos, machos y hembras. Cuatro hatos se asignaron al azar a cada uno de los dos tratamientos: en el primer tratamiento, el hato se manejó en grupos de 4-5 animales (TS), mientras que, en el segundo, se manejó en grupos de 10 a 12 animales (TG). El manejo consistió en llevar los animales a través de una manga de $13 \mathrm{~m}$ de longitud donde recibieron una aplicación subcutánea de Ivermectina al 1\%. Durante su paso por la manga, el tiempo promedio para manejar a los animales en cada tratamiento $(42.5 \pm 2.2$ vs $51.04 \pm 1.9 \mathrm{~min}$; $P<0.05)$, así como el número de animales que vocalizaron ( $5.5 \pm 0.6$ vs $7.7 \pm 0.2 ; \mathrm{P}<0.05)$, se 
regresaron (6.3 \pm 0.4 vs $9.5 \pm 0.6 ; P<0.05)$ y saltaron sobre sus compañeros $(2.7 \pm 0.5 v s 5.2$ $\pm 0.5 ; P<0.05$ ), fue menor en TS que en TG. Sin embargo, no se encontró diferencia entre los tratamientos $(P>0.05)$ para el número de animales que se golpearon (TS: $2.7 \pm 0.4$ y TG: $5.5 \pm 1.7$ ) o se cayeron (TS: $2 \pm 0.4$ y TG: $3.7 \pm 1.0$ ). Se concluye que el manejo de bovinos en grupos pequeños requiere menos tiempo y puede ser menos estresante y peligroso que cuando se manejan grupos más numerosos.

Palabras clave: Bienestar animal, Conducta, Instalaciones, Manga de manejo.

Recibido: 24/10/2018

Aceptado: 25/07/2019

En condiciones extensivas y semi-extensivas el ganado permanece en las áreas de pastoreo y sólo se conduce a los corrales para realizarles prácticas de manejo como marcaje, castración y medicina preventiva una o dos veces al año ${ }^{(1)}$. Este manejo no siempre se lleva a cabo en instalaciones adecuadas, ya que en numerosas ocasiones particularmente en el medio rural tropical se cuenta con infraestructura rústica deficiente, conformada por una manga de manejo recta y en ocasiones un corral, generalmente construidos con material de la región ${ }^{(2)}$. La falta de instalaciones impide el manejo de lotes homogéneos (tamaño, edad y sexo), y el manejo infrecuente impide la habituación del ganado a las instalaciones y a la presencia de operadores. Estos factores aunados a la falta de capacitación en los vaqueros ${ }^{(3)}$, favorecen la presencia de condiciones estresantes para los animales y un manejo agitado del hato, que pueden exponerles junto con los vaqueros a un riesgo de lesionarse muy elevado ${ }^{(4,5)}$. Estos factores también contribuyen a generar pérdidas económicas debidas a mala aplicación de tratamientos, lesiones de ganado, accidentes con el personal ${ }^{(6)}$, mermas de peso, fallas reproductivas o muertes, así como deterioro en la calidad de la carne ${ }^{(7)}$.

La presión de hacinamiento y el manejo de grupos grandes son algunas variables que en general incrementan la tensión y el estrés de los bovinos ${ }^{(8)}$. Los animales estresados, durante su manejo presentan alteraciones fisiológicas importantes que estimulan conductas como: orinar, defecar y salivar, así como: vocalizaciones, caídas, resbalones, golpes y brincos ${ }^{(9)}$. Por otra parte, animales en estas condiciones, eliminan feromonas que pueden ser percibidas por otros animales, provocándoles también alerta y estrés, evitando así que el ganado circule con facilidad ${ }^{(10)}$. En apoyo a lo anterior otros investigadores mencionan que el ganado tranquilo entra con facilidad al cajón de contención, pero si algún animal forcejea y se estresa, el resto de los animales se rehusará a ingresar con facilidad, dificultando el flujo del ganado $^{(11,12)}$.

El manejo de animales en grupos pequeños podría facilitar el flujo durante su paso por la manga, disminuyendo situaciones estresantes y comportamientos que puedan representar un 
riesgo, brindando a los animales la posibilidad de mantener un mejor estado de bienestar, permitiendo en consecuencia un manejo más eficiente y expedito. Por lo tanto, el objetivo del presente trabajo fue evaluar la velocidad de paso y la incidencia de algunas conductas relacionadas con el estrés, durante su manejo en grupos con diferente número de individuos, durante su paso por una manga recta para la aplicación de medicamento.

El trabajo se realizó con productores comerciales de ganado de carne en el estado de Guerrero, México $\left(18^{\circ} 25^{\prime} \mathrm{N}\right.$ y $\left.100^{\circ} 31^{\prime} \mathrm{W}\right)$ durante la época secas, bajo un clima cálido seco (AW0), con una temperatura de 35 a $40{ }^{\circ} \mathrm{C}$, humedad relativa de $25 \%$, vegetación selva baja caducifolia y $250 \mathrm{msnm}$. En esta región, el ganado se maneja una o dos veces por año y se cuenta con una manga recta y corral rústico que comparten diferentes ganaderos de la zona.

El manejo se realizó en una manga recta de 13 x $0.8 \mathrm{~m}$, construida con tubos de acero, postes de concreto, piso de tierra y sin sombra. La manga tenía una puerta corrediza del mismo material en un extremo, y se colocaba un tubo para impedir que el último animal del grupo se moviera hacia atrás. Los animales antes de entrar a la manga se alojaron durante 20 min en un corral de espera de $300 \mathrm{~m}^{2}$ con $50 \%$ de sombra, donde contaban con acceso libre al agua en un bebedero de 2.0 × 1.0 × $0.8 \mathrm{~m}$, para permitir su recuperación luego del traslado. El corral contaba también con un embudo para facilitar el ingreso hacia la manga. Al salir de la manga los animales se alojaron en otro corral muy similar al anterior, donde permanecieron hasta terminar el manejo de todo el hato, para después ser trasladados nuevamente a las áreas de pastoreo.

Se utilizaron ocho hatos comerciales, cada uno integrado por 50 bovinos cruza Brahman $\mathrm{x}$ Pardo Suizo en diferentes proporciones, criados en un sistema de manejo semi-extensivo. Cada hato estaba conformado con el $20 \%$ de animales entre 8 meses a 2 años de edad ( $\mathrm{n}=$ 10), $30 \%$ entre los 2 a 4 años $(n=15)$ y $50 \%$ de más de 4 años de edad $(n=25)$. En cada hato el $90 \%$ de los animales fueron hembras y el $10 \%$ machos.

El día de la evaluación, un hato se trasladaba a las 0600 h, del área de pastoreo hacia las instalaciones de manejo, mediante el arreo durante 30 o $40 \mathrm{~min}$, con un manejo tranquilo, hasta introducirlos en el corral de espera.

Se trabajó un hato diferente diariamente durante un periodo de ocho días continuos, aplicando los tratamientos de forma alternada y manejando los animales bajo las mismas condiciones durante todo el periodo de evaluación que comprendió del 13 al 20 de febrero de 2018.

Los ocho hatos se asignaron aleatoriamente a uno de dos tratamientos; Sistemas de manejo en grupos pequeños (TS) o en grupos más numerosos (TG). En el TS, se manejaban grupos integrados en forma aleatoria por cuatro a cinco animales, que se llevaban a la manga, y se les aplicaba el medicamento. Posteriormente se removía el ganado de la manga y se repetía 
el procedimiento hasta completar el manejo de todo el hato. En TG el manejo era similar, pero el hato se trabajaba en grupos de 10 a 12 animales, que era la capacidad máxima de la manga.

El manejo se realizó de una manera tranquila con la ayuda de cuatro personas con experiencia en el manejo de animales. Un médico veterinario aplicó Ivermectina subcutánea al 1\% mientras estos permanecían en la manga, y una persona experimentada previamente entrenada se dedicó a la observación de los animales, registrando los tiempos y el comportamiento con base en el etograma que se describe en el Cuadro 1.

Cuadro 1: Etograma de las conductas evaluadas durante el manejo de bovinos a través de una manga recta cuando este se realizó con grupos reducidos de animales o en grupos más numerosos

\begin{tabular}{ll}
\hline Variable & Medición \\
\hline Vocalizar & $\begin{array}{l}\text { Animales que emitieron sonidos o llamados, originados desde sus } \\
\text { gargantas y hocicos. } \\
\text { Animales que perdieron el apoyo sobre sus extremidades y su } \\
\text { cuer }\end{array}$ \\
Animales que golpearon o trataron de golpear a otros animales, \\
Golpes & $\begin{array}{l}\text { Anueros o las instalaciones, con las patas, cabeza o atropellando. } \\
\text { Animales que avanzaron o trataron de avanzar pasando por encima } \\
\text { Brincar }\end{array}$ \\
Regresar & $\begin{array}{l}\text { Animales que se dieron vuelta dentro de la manga tratando de } \\
\text { circular en sentido contrario al flujo. }\end{array}$ \\
\hline
\end{tabular}

Durante la evaluación sólo se consideró el número de animales que realizaron las conductas registradas, independientemente de la frecuencia con que estas se realizaron.

El tiempo invertido en el manejo de los animales se analizó mediante una prueba de " $t$ " de Student, comparando los resultados de TS vs TG. Cada hato fue considerado como una repetición dentro de cada tratamiento, y cada animal constituyó la unidad experimental. Las variables de comportamiento entre los dos tratamientos se compararon mediante la prueba de Mann Whitney. Finalmente, se realizó una correlación de rangos Tau de Kendall entre las variables: tiempo y número de animales que vocalizan, caen, golpean, brincan y regresan.

El tiempo promedio requerido para manejar los 50 animales fue menor en TS que en TG 42.5 \pm 2.2 min vs $51.04 \pm 1.9$ min; $P<0.05$; respectivamente. Además, menos animales vocalizaron, se voltearon y brincaron encima de otros en TS en comparación con TG $(5.5 \pm$ 0.6 vs $7.7 \pm 0.2 ; 6.3 \pm 0.4$ vs $9.5 \pm 0.6$ y $2.7 \pm 0.5$ vs $5.2 \pm 0.5$, respectivamente; Figura 1 ). 
No se encontraron diferencias entre tratamientos $(P>0.05)$ en el número de animales que cayeron al suelo ni en animales que golpearon. El tiempo invertido en el manejo mostró una correlación significativa $(\mathrm{r}=0.56$ a $0.79 ; P<0.01)$ con el número de animales que vocalizan, caídos, golpean, brincan y regresan (Cuadro 2).

Figura 1: Promedio ( $\pm \mathrm{EE})$ de tiempo de manejo y número de animales que muestran algunos comportamientos que indican estrés en bovinos manejados en grupos (TG) y grupos (TS) en una manga recta
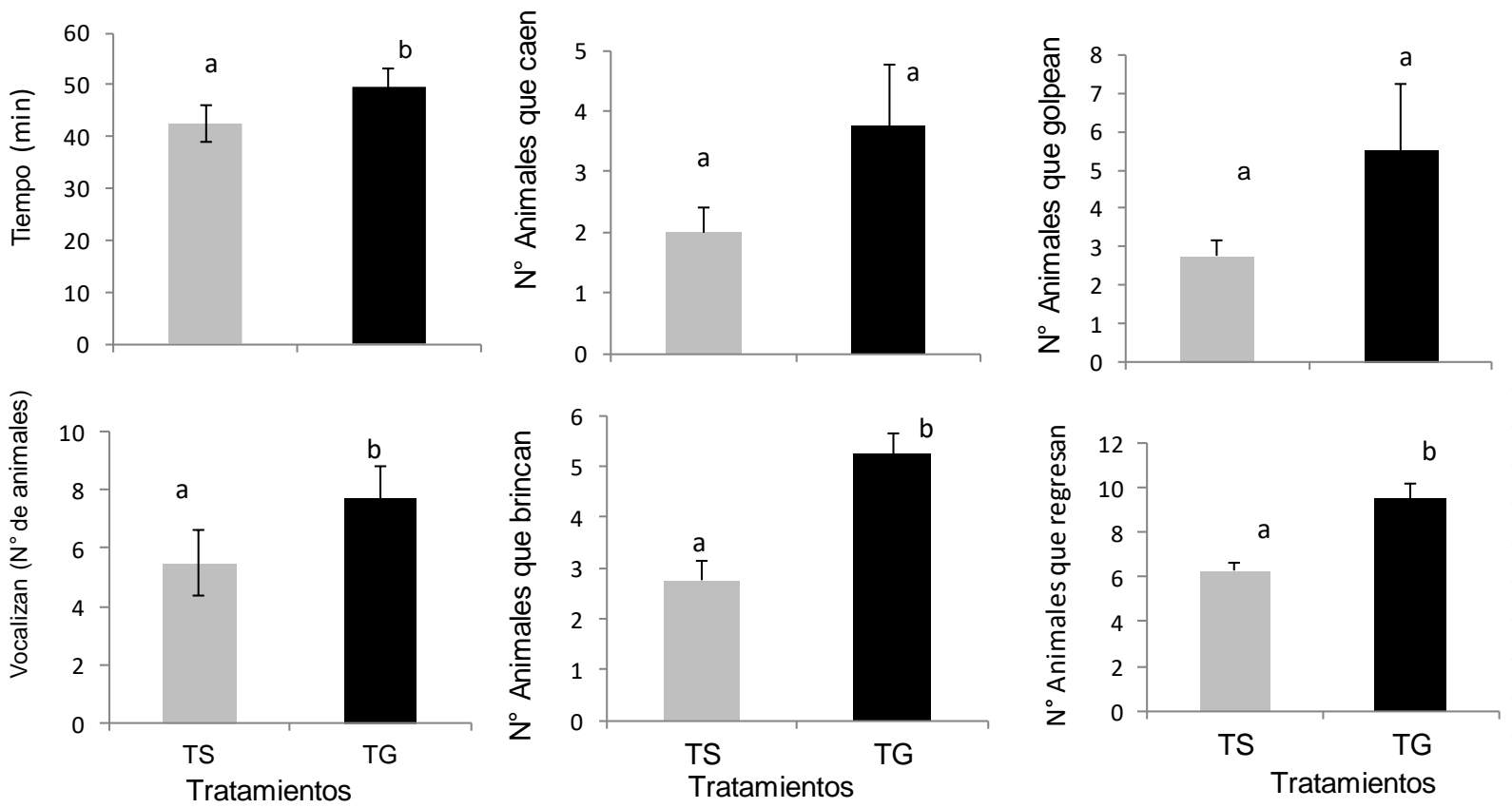

${ }^{\mathrm{ab}}$ Diferente literal indica diferencia $(P<0.05)$.

Cuadro 2: Correlación de rangos (TAU DE KENDALL) de algunas variables que indican estrés en bovinos manejados bajo diferentes tamaños de grupos a través de una manga recta

\begin{tabular}{llllll}
\hline & Vocalizan & Caen & Golpean & Brincan & Regresan \\
\hline Tiempo & $0.677 * *$ & $0.624 * *$ & $0.562 * *$ & $0.790 * * *$ & $0.717 * * *$ \\
Vocalizan & & $0.733 * * *$ & $0.699 * *$ & $0.758 * * *$ & $0.794 * * *$ \\
Caen & & & $0.760 * * *$ & $0.803 * * *$ & $0.678 * *$ \\
Golpean & & & & $0.688^{* *}$ & $0.740 * * *$ \\
Brincan & & & & & $0.812 * * *$ \\
\hline
\end{tabular}

$*(P<0.05), * *(P<0.01), * * *(P<0.001)$. 
$\mathrm{Al}$ reducir el tamaño de los grupos se logra un manejo más eficiente y seguro para animales y vaqueros. El número de animales que realiza las conductas evaluadas está altamente relacionado con el tiempo de manejo. Lo anterior podría significar que, al requerir más tiempo para el arreo de grupos más grandes, éste ocasiona que más animales realicen conductas agresivas, quizá debido a periodos mayores de exposición a climas extremos, en contacto con animales invadiendo sus espacios individuales o dentro de instalaciones y con operadores que no les son familiares. $\mathrm{O}$, por otro lado, que al haber un mayor número de animales realizando estas conductas, esto provoque que el paso de los animales por las instalaciones sea más tardado. Los resultados de este trabajo coinciden con los estudios realizados por otros investigadores, quienes indican que los animales presentan más conductas agresivas cuando el tiempo de contención es mayor ${ }^{(13,14)}$.

Paralelamente, el manejo de grupos reducidos podría ser un sistema más seguro, al observar que un menor número de animales realiza conductas que les ponen en riesgo de lesionarse durante su paso por la manga, disminuyendo el riesgo de accidentes entre los animales y hacia los vaqueros.

El tamaño del grupo fue factor determinante para encontrar diferencia entre los tratamientos. El llenar la manga de manejo a su máxima capacidad con animales de diferente talla, edad y sexo, pudo provocar invasión del espacio individual de un mayor número de animales, así como favorecer que más animales sumisos fueran forzados a permanecer cerca de animales dominantes, propiciando así un mayor número de animales manifestando conductas indicativas de estrés.

En un hato establecido, existe relaciones de dominancia sumisión bien establecidas, que permiten la coexistencia de los animales ${ }^{(15)}$, por lo que cuando se les fuerza a interactuar con otros animales, incrementando su densidad y creando nuevos grupos de diferente rango de edad y peso, estos son factores clasificados como estresores bilógicos ${ }^{(16)}$. Así mismo, estas prácticas se relacionan con una mala calidad en el manejo del ganado y se asocian con una mayor reactividad, más comportamientos indeseables y un mayor riesgo de accidentes ${ }^{(17,18)}$. En suma toda situación que rompa con la organización social en una población animal puede desencadenar diferentes grados de estrés ${ }^{(14,19)}$, esta situación fue propiciada en el presente estudio al provocar un cambio en el entorno espacial durante el llenado de la manga de manejo.

Por otra parte, existe la posibilidad de que, al manejar grupos mayores, los últimos animales del grupo puedan percibir mayor cantidad de señales de estrés, porque frente a ellos hay una mayor cantidad de animales en estrés, reduciendo su velocidad de flujo. Esta misma situación podría presentarse al requerirse más tiempo en el manejo de mayor cantidad de animales, lo que también favorecería la emisión de señales de estrés, tanto conductuales como químicas, y la consecuente precepción de las mismas por los últimos animales de cada grupo ${ }^{(20)}$. En ganado Bos taurus, Grandin $^{(21,22)}$ y Enríquez et al ${ }^{(23)}$ encontraron resultados similares, 
observando que grupos pequeños pueden dirigirse hacia la sala de matanza con mayor facilidad, provocando menos vocalizaciones, resbalones y caídas en los grupos.

Con base en los resultados del presente experimento, se observa que el manejo de animales en TS puede ser un sistema alternativo y simple, que reduce las conductas indicativas de estrés sin generar un costo extra, y aprovechando la infraestructura mínima existente en la mayoría de los ranchos donde se maneja el ganado en condiciones semi-extensivas tropicales.

El manejo de bovinos en grupos pequeños requiere menos tiempo, y menos animales realizan conductas indicativas de estrés durante su manejo en una manga recta, por lo que se considera que este sistema es más eficiente y proporciona un mayor grado de bienestar animal.

\section{Conflicto de intereses}

Los autores declaran no tener conflicto de intereses en relación con el presente trabajo

\section{Agradecimientos}

A los dueños de los hatos por su disposición para realizar el trabajo en sus unidades de producción y a los vaqueros que colaboraron para el manejo del ganado.

Al Consejo Nacional de Ciencia y Tecnología (CONACYT) por el otorgamiento de beca para realizar estudio de Doctorado.

\section{Literatura citada:}

1. Garcia MA, Albarran PB, Avilez NF. Dynamics and trends in dual purpose cattle management in southern Estado de México. Agrociencia 2015;9(2):125-139.

2. Nájera GA, Piedra MR, Albarrán PB, García MA. Changes in dual purpose livestock farming system in the dry tropic of Estado de Mexico. Agrociencia 2016;50(6):701-710. 
3. Sant'Anna CA, Paranhos da Costa MJR. Como as práticas de bea podem mehorar a bovinocultura moderna. Simpósio da Ciência do Bem-estar Anima. Escola de veterinária da UFMG Belo Horizote MG, Brazil. 2009:42-47.

4. Breuer K, Hemsworth PH, Barnett JL, Matthews LR, Coleman GR. Behavioral response to humans and the productivity of commercial dairy cows. Appl Anim Behav Sci 2000;66(4):273-288.

5. Sutherland AM, Dowling KS. The relationship between responsiveness of first-lactation heifers to humans and the behavioral response to milking and milk production measures. J Vet Behav 2014;9(1):30-33.

6. Paranhos da Costa MJR, Macedo de Toledo L, Schidek A. Boas Pácticas de manejo, Vacinação. 1a ed. Jaboticabal, Brazil: Livraria \& Editora Funep; 2006.

7. Gallo C, Tadich N. Transporte terrestre de bovinos: efectos sobre el bienestar animal y la calidad de la carne. Agrociencia 2005;21(2):37-49.

8. Costa A, Dasso L. Manejo de bovinos en sistemas productivos: Caracterización de dos estilos de manejo y niveles sanguíneos de cortisol. Red Vet 2007;8(1):1695-7504.

9. Vieuille TC, Signoret JP. Pheromonal transmission of an aversive experience in domestic pig. J Chem Ecol 1992;18(9):1551-1557.

10. Grandin T. Assessment of stress during handling and transport. J Anim Sci 1997;75(1):249-257.

11. Muñoz D, Strappini A, Gallo C. Indicators of animal welfare to detect problems in the box of desensitization of bovines. Arch Vet Med 2012;44(3):297-302.

12. Romero PM, Sanchez VJ. Animal welfare during transport and its relationship with meat quality. J MVZ Cordoba 2012;17(1):2936-2944.

13. Burdick NC, Rundel RD, Carroll JA, Welsh Jr TH. Interaction between temperament, stress, and immune function in cattle. Int J Zool 2011;20(11):1-9.

14. Proudfoot K, Habing G. Social stress as a cause of diseases in farm animals: current knowledge and future directions. Vet J 2015;206(1):15-21.

15. Fukasawa M, Tsakada H. Relationship between milk cortisol concentration and the behavioral characteristics of postpartum cows introduced to a new group. Anim Sci J 2010;81(5):612-617.

16. Asres A, Amha N. Efect of stress on animal health: a review. J Biol Agric Health 2004;4(27):116-121. 
17. Ceballos MC, Sant'Anna AC, Góis KCR, Ferraudo AS, Negrao JA, Paranhos da Cosdta MJR. Investigating the relation-ship between human-animal interactions, reactivity, stress response and reproductive performance in Nellore heifers. Livest Sci 2018;217(1):65-75.

18. Ceballos MC, Sant'Anna AC, Boivin X, Oliveira Costa FO, Carvalhal MV de L, Paranhos da Costa MJR. Impact of good practices of handling training on beef cattle welfare and stock people attitudes and behaviors. Livest Sci 2018;216(1):24-31.

19. Enríquez D, Hotzel M, Ungerfeld R. Minimizing the stress of beef calves: a review. Acta Vet Scand 2011;53(1):2-8.

20. Orihuela JA, Solano JJ. Relationship between order of entry in slaughterhouse raceway and time to traverse raceway. Appl Anim Behav Sci 1994;40(3):313-317.

21. Grandin T, Deesing MJ, Struthers JJ, Swinker AM. Cattle with hair whorl patterns above the eyes are more behaviorally agitated during restraint. Appl Anim Behav Sci 1995;46(1-2):117-123.

22. Grandin T. Improving animal welfare: A practical approach. 2nd ed. Wallingford, UK: CABI International; 2015.

23. Enríquez DH, Ungerfeld R, Quintans G, Guidoni AL, Hotzel MG. The effects of alternative weaning methods on behaviour in beef calves. Livest Sci 2010;128(1-3): 20-

27. 